\title{
Hypoplastic Cam-clay model
}

David Mašín

Charles University in Prague

corresponence address:

David Mašín

Charles University in Prague

Faculty of Science

Albertov 6

12843 Prague 2, Czech Republic

E-mail:masin@natur.cuni.cz

Tel: +420-2-2195 1552, Fax: +420-2-21951556

January 30, 2012

Number of words: 1974 (excluding abstract, references, acknowledgement and figure captions)

Number of tables: 1

Number of figures: 4

Revised version of a Technical Note for Géotechnique 


\section{Abstract}

A rearrangement of the hypoplastic constitutive equation is proposed that enables the incorporation of an asymptotic state boundary surface of an arbitrary pre-defined shape into the model, with any corresponding asymptotic strain rate direction. This opens the way for further development of hypoplastic models. To demonstrate the flexibility of the proposed approach, a hypoplastic equivalent of the Modified Cam-clay model is developed. A comparison of the predictions of the elasto-plastic and hypoplastic models reveals the merits of the hypoplastic formulation. While both models predict the same asymptotic states, hypoplasticity predicts a smooth transition between overconsolidated and normally consolidated states, and thus accounts for the non-linearity of the soil behaviour inside the state boundary surface in a natural manner.

Keywords: hypoplasticity; Cam-clay; asymptotic states; critical state; state boundary surface

\section{Introduction}

Over the past two decades, hypoplasticity has proven to be a powerful approach to predict the nonlinear behaviour of soils. Early hypoplastic models were developer from sound physical assumptions by means of trial-and-error procedures (see Kolymbas 1991). These models represented the soil behaviour reasonably well, but their parameters did not have any clear physical meaning.

Throughout the development of hypoplasticity, several milestones can be identified, and these are often related to predictions of asymptotic states. An asymptotic state is that reached asymptotically after sufficiently long stretching with a constant strain rate direction (see Gudehus and Mašín 2009). An envelope of asymptotic states plotted in the stress vs. void ratio space forms a socalled asymptotic state boundary surface (ASBS) (Mašín 2012). In elasto-plastic models, such as the Modified Cam-clay model (Roscoe and Burland 1968), the ASBS coincides with the state boundary surface. It is, in turn, formed by the combination of the yield surface and a hardening law.

The first advance in incorporating asymptotic states into hypoplasticity was attributed to Gudehus (1996) and Bauer (1996). They realised that a certain combination of constants in the early model caused it to asymptotically predict the shear failure at a constant volume. They also introduced normal compression and critical state lines into the model, thus incorporating the critical state behaviour. von Wolffersdorff (1996) then modified the model so that the critical state locus coincided exactly with the condition proposed by Matsuoka and Nakai (1974). Later, Niemunis (2002) put forward a general approach to incorporate an arbitrary pre-defined critical state locus into hypoplasticity. His procedure was adopted by Mašín (2005) as a basis for developing a model for clays. Subsequently, Mašín and Herle (2005) proposed a way to extract the complete shape of the ASBS from the hypoplastic equation. Its shape was shown to depend on the material parameters, while only isotropic and critical asymptotic states were pre-defined. The complete shape of the ASBS thus could not be prescribed $a$ priori, as is normally done in elasto-plastic models. This property of hypoplasticity has been regarded as its serious limitation.

In this Note, the next step in the development of hypoplastic models is presented. It is shown that the hypoplastic equation can be,rearranged, in a relatively straightforward manner so that the model predicts any arbitrary pre-defined shape of ASBS and asymptotic strain rate direction. To demonstrate the proposed approach, a hypoplastic model with ASBS and a flow rule of the Modified Cam-clay model is developed.

Notations and Conventions: $\|\mathrm{X}\|$ represents the Euclidean norm of $\mathrm{X}$, defined as $\|\mathrm{X}\|=\sqrt{\mathrm{X}: \mathrm{X}}$. The trace operator is defined as $\operatorname{tr} \mathrm{X}=1: \mathrm{X} ; 1$ and $I$ denote second- and fourth-order unity tensors, respectively. Following the sign convention of continuum mechanics, compression is taken as negative. However, 
the mean stress $p=-\operatorname{tr} \sigma / 3$ is defined as positive in compression. $\sigma^{*}$ represents the stress deviator $\sigma^{*}=\sigma+1 p$.

\section{Explicit incorporation of an asymptotic state boundary surface into hypoplasticity}

A general formulation of the hypoplastic model may be written as (Gudehus 1996)

$\dot{\boldsymbol{\sigma}}=f_{s}\left(L: \dot{\boldsymbol{\epsilon}}+f_{d} \mathbf{N}\|\dot{\boldsymbol{\epsilon}}\|\right)$

where $\dot{\boldsymbol{\sigma}}$ and $\dot{\boldsymbol{\epsilon}}$ are the stress and strain rate tensors respectively ${ }^{1}, L$ and $\mathbf{N}$ are fourth- and second-order constitutive tensors, $f_{s}$ is the factor controlling the influence of mean stress (barotropy factor) and $f_{d}$ is the factor controlling the influence of relative density (pyknotropy factor).

To evaluate the model response at the ASBS, we will interpret it in the stress space normalised by the size of the constant void ratio cross-section through the ASBS. It is given by the Hvorslev equivalent pressure $p_{e}^{*}$, defined as a mean stress at the isotropic normal compression line at the current void ratio $e$. The normalised stress thus reads $\sigma_{n}=\sigma / p_{e}^{*}$ and it follows that

$\dot{\boldsymbol{\sigma}}_{n}=\frac{\dot{\boldsymbol{\sigma}}}{p_{e}^{*}}-\frac{\sigma}{p_{e}^{* 2}} \dot{p}_{e}^{*}$

The following derivations are for normal compression lines linear in the $\ln (1+e)$ vs. $\ln \left(p / p_{r}\right)$ plane ( $p_{r}$ is the reference stress of $1 \mathrm{kPa}$ ), but other expressions may also be introduced. The isotropic normal compression line can be written as

$\ln (1+e)=N-\lambda^{*} \ln \left(p_{e}^{*} / p_{r}\right)$

where $N$ and $\lambda^{*}$ are model parameters. It follows that

$p_{e}^{*}=p_{r} \exp \left[\frac{N-\ln (1+e)}{\lambda^{*}}\right]$

and thus

$\dot{p}_{e}^{*}=-\frac{p_{e}^{*}}{\lambda^{*}}\left(\frac{\dot{e}}{1+e}\right)=-\frac{p_{e}^{*}}{\lambda^{*}} \operatorname{tr} \dot{\boldsymbol{\epsilon}}$

Combining (5), (2) and (1) implies that

$\dot{\boldsymbol{\sigma}}_{n}=\frac{f_{s}}{p_{e}^{*}}\left(\boldsymbol{L}: \dot{\boldsymbol{\epsilon}}+f_{d} \mathbf{N}\|\dot{\boldsymbol{\epsilon}}\|\right)+\frac{\boldsymbol{\sigma}}{p_{e}^{*} \lambda^{*}} \operatorname{tr} \dot{\boldsymbol{\epsilon}}$

During asymptotic stretching the stress state remains fixed in the $\boldsymbol{\sigma}_{n}$ space (Mašín and Herle 2005), provided that the constant void ratio cross-sections through the ASBS differ only in size and not in shape. This condition implies $\dot{\boldsymbol{\sigma}}_{n}=\mathbf{0}$. It then follows from (2) that

$-\frac{\sigma}{\lambda^{*}} \operatorname{tr} \dot{\boldsymbol{\epsilon}}^{A}=f_{s}\left(L: \dot{\boldsymbol{\epsilon}}^{A}+f_{d}^{A} \mathbf{N}\left\|\dot{\boldsymbol{\epsilon}}^{A}\right\|\right)$

where $f_{d}^{A}$ is the value of $f_{d}$ at the ASBS and $\dot{\boldsymbol{\epsilon}}^{A}$ is the asymptotic strain rate corresponding to the given stress state. Eq. (7) can be manipulated in the following way:

\footnotetext{
${ }^{1}$ To be precise, $\dot{\boldsymbol{\sigma}}$ represents the objective (Jaumann) stress rate and $\dot{\boldsymbol{\epsilon}}$ is the Euler stretching tensor.
} 
$-\left(\frac{\sigma}{\lambda^{*}} \operatorname{tr} \dot{\boldsymbol{\epsilon}}^{A}+f_{s} L: \dot{\boldsymbol{\epsilon}}^{A}\right)=f_{s} f_{d}^{A} \mathbf{N}\left\|\dot{\boldsymbol{\epsilon}}^{A}\right\|$

$-\boldsymbol{A}: \dot{\boldsymbol{\epsilon}}^{A}=f_{s} f_{d}^{A} \mathbf{N}\left\|\dot{\boldsymbol{\epsilon}}^{A}\right\|$

$-\boldsymbol{A}: \boldsymbol{d}=f_{s} f_{d}^{A} \mathbf{N}$

where

$A=f_{S} L+\frac{\sigma}{\lambda^{*}} \otimes 1$

$\boldsymbol{d}=\frac{\dot{\boldsymbol{\epsilon}}^{A}}{\left\|\dot{\boldsymbol{\epsilon}}^{A}\right\|}$

Eq. (10) implies that

$\mathbf{N}=-\frac{A: \boldsymbol{d}}{f_{s} f_{d}^{A}}$

Combining (13) with (1) yields an alternative expression of the hypoplastic model

$\dot{\boldsymbol{\sigma}}=f_{s} L: \dot{\boldsymbol{\epsilon}}-\frac{f_{d}}{f_{d}^{A}} A: \boldsymbol{d}\|\dot{\boldsymbol{\epsilon}}\|$

An arbitrary shape of the ASBS can be incorporated into hypoplasticity with the aid of Eq. (14), by appropriate specification of the dependency of $f_{d}^{A}$ on the void ratio and stress ratio. The corresponding asymptotic direction of the strain rate is then prescribed by $\boldsymbol{d}$. This can be done independently of the selected expression for the tensor $L$.

\section{Hypoplastic Cam-clay model}

To demonstrate development of a hypoplastic model using Eq. (14), this section will outline the formulation of a hypoplastic equivalent of the Modified Cam-clay model. Slight modifications to the original elasto-plastic formulation are introduced to maximise the model simplicity, and are not generally required. In particular, normal compression lines linear in the $\ln (1+e)$ vs. $\ln \left(p / p_{r}\right)$ plane (Butterfield 1979) were considered. In the Modified Cam-clay model, the ASBS is elliptic in the $p$ vs. $q$ plane for a constant preconsolidation pressure $p_{c}$. On the other hand, in the hypoplastic formulation, the ASBS is elliptic for constant $p_{e}^{*}$. Finally, the asymptotic total strain rate tensor is assumed to be normal to the ASBS in the hypoplastic model, while in the elasto-plastic case the associated flow rule implies that the plastic strain rate tensor is normal to the ASBS.

The tensor $L$ is represented by isotropic elasticity, i.e.

$L=I+\frac{v}{1-2 v} \mathbf{1} \otimes \mathbf{1}$

where the parameter $v$ controls the proportion of bulk and shear stiffness. Effectively, it regulates the shear stiffness, since the bulk stiffness in the model is controlled by the parameters $\lambda^{*}$ and $\kappa^{*}$ (as shown later).

The following expression for the factor $f_{d}$, which governs the non-linear behaviour inside the state boundary surface, is chosen:

$f_{\boldsymbol{d}}=\frac{2 p}{p_{e}^{*}}$ 
Note that the model formulation is not restricted to the particular form given by (16), and different formulations can also be used. In the $p$ vs. $q$ plane the ASBS has an elliptic shape, prescribed by the yield function $f$ of the Modified Cam-clay model (with $p_{e}^{*}$ in place of $p_{c}$ ):

$f=0=q^{2}+M^{2} p^{2}-M^{2} p p_{e}^{*}$

where $M$ is the slope of the critical state line in the $p$ vs. $q$ plane and $q=\sqrt{3 / 2}\left\|\sigma^{*}\right\|$. It follows from Eq. (17) that for the given stress ratio $\eta=q / p$, the value of mean stress at the ASBS $p^{A}$ is

$p^{A}=p_{e}^{*} \frac{M^{2}}{M^{2}+\eta^{2}}$

$f_{d}^{A}$ is then expressed as $f_{d}^{A}=2 p^{A} / p_{e}^{*}$ (from (16)), and the ratio $f_{d} / f_{d}^{A}$ needed in Eq. (14) reads as

$\frac{f_{d}}{f_{d}^{A}}=\frac{p}{p^{A}}=\left(\frac{p}{p_{e}^{*}}\right) \frac{M^{2}+\eta^{2}}{M^{2}}$

The asymptotic strain rate direction $\boldsymbol{d}$ is assumed to be normal to the ASBS, following the Modified Cam-clay formulation, although any other direction can be adopted instead. In the present case,

$\boldsymbol{d}=\frac{\partial g / \partial \boldsymbol{\sigma}}{\|\partial g / \partial \boldsymbol{\sigma}\|}$

where $g=f$. It follows from Eq. (17) that $\partial g / \partial \sigma$ at the ASBS at current stress ratio $\sigma^{*} / p$ reads

$\frac{\partial g}{\partial \sigma}=3 \frac{\sigma^{*}}{p} p^{A}-1 \frac{M^{2}}{3}\left(2 p^{A}-p_{e}^{*}\right)$

Combination of (21) with (18) yields

$\boldsymbol{d}=\frac{3 \boldsymbol{\sigma}^{*}-1 p\left(M^{2}-\eta^{2}\right) / 3}{\left\|3 \boldsymbol{\sigma}^{*}-1 p\left(M^{2}-\eta^{2}\right) / 3\right\|}$

The last component of the model to be defined is the factor $f_{s}$. It is specified to ensure that the slope of the isotropic unloading line in the $\ln (1+e)$ vs. $\ln p / p_{r}$ plane, for unloading starting from the isotropic normally consolidated state, is given by $\kappa^{*}$. Note that the slope $\lambda^{*}$ of the isotropic normal compression line is already implicit in the model formulation, since it has been adopted as a primary assumption in the derivation of the tensor $\boldsymbol{A}$. Algebraic manipulations with the above tensorial equations reveal that for unloading (volume increase, $\dot{e}>0$ ), the isotropic form of the model is given as:

$\dot{p}=\left[\frac{p}{\lambda^{*}}-2 f_{S}\left(\frac{1}{3}+\frac{v}{1-2 v}\right)\right] \frac{\dot{e}}{1+e}$

Eq. (23) can be compared with $e^{\cdot} /(1+e)=-\kappa^{*} p^{*} / p$, which leads to an expression for $f_{s}$

$f_{S}=\frac{3 p}{2}\left(\frac{1}{\lambda^{*}}+\frac{1}{\kappa^{*}}\right) \frac{1-2 v}{1+v}$

A complete formulation of the hypoplastic Cam-clay model can finally be written as

$\dot{\boldsymbol{\sigma}}=f_{S} L: \dot{\boldsymbol{\epsilon}}-\left(\frac{p}{p_{e}^{*}}\right) \frac{M^{2}+\eta^{2}}{M^{2}}\left(f_{S} L+\frac{\boldsymbol{\sigma}}{\lambda^{*}} \otimes \mathbf{1}\right): \boldsymbol{d}\|\dot{\boldsymbol{\epsilon}}\|$

with $f_{s}$ given by (24), $L$ by (15), $\boldsymbol{d}$ by (22) and $p_{e}^{*}$ by (4). 


\section{Model predictions}

In this section, predictions by the proposed hypoplastic model are compared with predictions obtained using the elasto-plastic Modified Cam-clay model ${ }^{2}$. The model parameters are given in Table 1. Different values of parameters $v$ were adopted in the two models to predict comparable stiffness in shear.

[Table 1 about here.]

Figure 1 shows constant void ratio cross-sections through the ASBSs of the two models and the response envelopes for different states. For details on the representation of tangential stiffness using response envelopes see Gudehus and Mašín (2009). As was explained in that paper, and as is clear from Fig. 1, the response envelopes of hypoplasticity are translated ellipses, whereas response envelopes of elasto-plasticity are at the ASBS composed of two elliptic sections centered about the reference state.

[Figure 1 about here.]

In Figure 2, predictions of drained triaxial tests are shown. All the samples were tested at the same void ratio $e=0.46$, but at different cell pressures (different overconsolidation ratios). Hypoplasticity and elasto-plasticity yield similar asymptotic large-strain predictions. However, before reaching the peak strength, hypoplasticity, unlike elasto-plasticity, predicts a non-linear response inside the ASBS with gradually decreasing stiffness. It also predicts a lower peak strength at the overconsolidated state than the elasto-plastic model. Hypoplastic formulation thus effectively eliminates two major shortcomings of the Modified Cam-clay model.

[Figure 2 about here.]

Figure 3a shows predictions of an isotropic loading and unloading test with several unloading/reloading cycles. The two models predict the same slope and position of the isotropic normal compression line, and the same slope of the unloading line at the isotropic normally consolidated state. In addition, hypoplasticity also predicts the non-linear response inside the ASBS. It does not predict the hysteretic unloading/reloading behavior, however. This shortcoming can be eliminated by adopting an enhancement introduced by Niemunis and Herle (1997). Fig. 3b shows $p$ vs. $q$ stress paths obtained in cyclic undrained triaxial tests. While elasto-plasticity predicts a stress path with constant $p$ inside the ASBS, hypoplasticity yields stress paths with a butterfly-like shape. The final (critical) state is practically the same in both cases (the difference seen is only attributed to subtle differences in the asymptotic state formulations of the two models).

[Figure 3 about here.]

Finally, Fig. (4) shows results of proportional compression tests (tests with constant direction of $\dot{\boldsymbol{\epsilon}}$ ). The tests differ in the value of the angle $\psi_{\dot{\epsilon}}$, which has been defined by Gudehus and Mašín (2009). $\psi_{\dot{\epsilon}}=0^{\circ}$ represents isotropic compression, whereas $\psi_{\dot{\epsilon}}=90^{\circ}$ represents constant volume (undrained) compression. Although the final (asymptotic) states predicted by the two models are similar, hypoplasticity again predicts a smoother transition between the overconsolidated and normally consolidated response.

[Figure 4 about here.]

\footnotetext{
${ }^{2}$ A modification of the model adopting normal compression line by Eq. (3) has been used in simulations.
} 


\section{Conclusions}

A new approach for the incorporation of the asymptotic state boundary surface of an arbitrary shape into hypoplastic models has been proposed. Unlike in the existing hypoplastic models, the ASBS can now be defined explicitly, and it is independent of the adopted expression for the tensor $L$. To demonstrate the proposed approach, a hypoplastic equivalent of the Modified Cam-clay model was developed. A comparison of the predictions of the elasto-plastic and hypoplastic models reveals several advantages of using the hypoplastic formulation. It predicts the non-linear response inside the ASBS and shows a gradual transition between normally consolidated and overconsolidated states. The proposed approach opens the way for further development of hypoplastic models.

\section{Acknowledgment}

Financial support by the research grants GACR P105/12/1705, GACR P105/11/1884, TACR TA01031840 and MSM 0021620855 is greatly appreciated.

\section{References}

Bauer, E. (1996). Calibration of a comprehensive constitutive equation for granular materials. Soils and Foundations 36 (1), 13-26.

Butterfield, R. (1979). A natural compression law for soils. Géotechnique 29 (4), 469-480.

Gudehus, G. (1996). A comprehensive constitutive equation for granular materials. Soils and Foundations $36(1), 1-12$.

Gudehus, G. and D. Mašín (2009). Graphical representation of constitutive equations. Géotechnique $52(2), 147-151$.

Kolymbas, D. (1991). Computer-aided design of constitutive laws. International Journal for Numerical and Analytical Methods in Geomechanics 15, 593-604.

Matsuoka, H. and T. Nakai (1974). Stress-deformation and strength characteristics of soil under three different principal stresses. In Proc. Japanese Soc. of Civil Engineers, Volume 232, pp. 59-70.

Mašín, D. (2005). A hypoplastic constitutive model for clays. International Journal for Numerical and Analytical Methods in Geomechanics 29 (4), 311-336.

Mašín, D. (2012). Asymptotic behaviour of granular materials. Granular Matter (submitted).

Mašín, D. and I. Herle (2005). State boundary surface of a hypoplastic model for clays. Computers and Geotechnics 32 (6), 400-410.

Niemunis, A. (2002). Extended Hypoplastic Models for Soils. Habilitation thesis, Ruhr-University, Bochum.

Niemunis, A. and I. Herle (1997). Hypoplastic model for cohesionless soils with elastic strain range. Mechanics of Cohesive-Frictional Materials 2, 279-299.

Roscoe, K. H. and J. B. Burland (1968). On the generalised stress-strain behaviour of wet clay. In J. Heyman and F. A. Leckie (Eds.), Engineering Plasticity, pp. 535-609.

Cambridge: Cambridge University Press. 
von Wolffersdorff, P. A. (1996). A hypoplastic relation for granular materials with a predefined limit state surface. Mechanics of Cohesive-Frictional Materials 1, 251-271.

\section{List of Figures}

1. Constant void ratio cross-sections through the asymptotic state boundary surfaces and response envelopes predicted by the two models. (a) hypoplasticity, (b) elasto-plasticity. $\sigma_{1}$ and $\sigma_{2}$ are axial and radial stresses respectively.

2. Predictions of drained triaxial tests for the same void ratio and different radial stresses (labels for cell pressures).

3. Comparison of predictions by the two models. (a) Isotropic test with several unloading/reloading cycles and (b) cyclic undrained triaxial test.

4. Proportional compression (constant direction of $\dot{\boldsymbol{\epsilon}}$ ) on initially overconsolidated soil. Values of $\psi_{\dot{\epsilon}}$ as defined by Gudehus and Mašín (2009) are indicated. Only selected paths are shown in (b) for clarity.

\section{List of Tables}

1. Parameters of used in the simulations. Parameter $v$ calibrated to predict comparable shear stiffness ( $v=0.2$ for the hypoplastic model and $v=0.32$ for the elasto-plastic model).

\begin{tabular}{|ccccc|}
\hline$M$ & $\lambda^{*}$ & $\kappa^{*}$ & $N$ & $v$ \\
1 & 0.1 & 0.01 & 1 & 0.2 or 0.32 \\
\hline
\end{tabular}




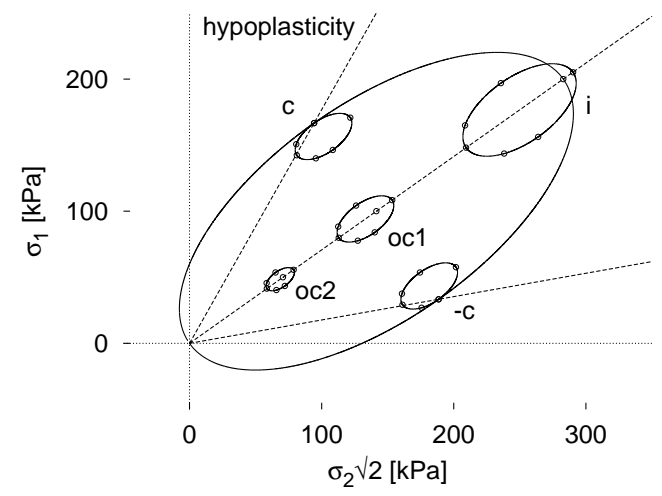

(a)

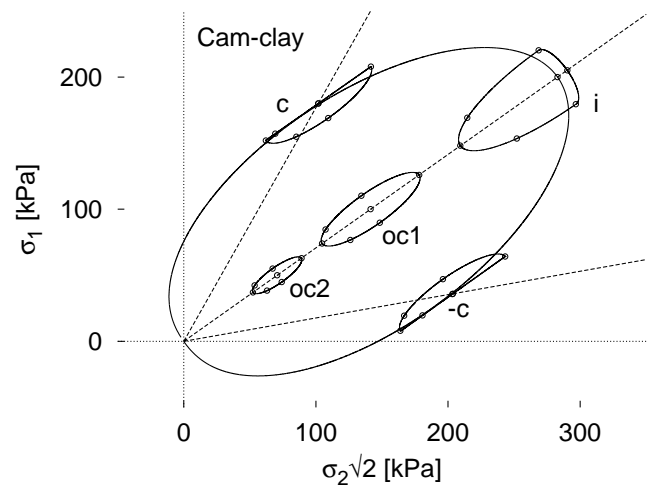

(b)

Figure 1: Constant void ratio cross-sections through the asymptotic state boundary surfaces and response envelopes predicted by the two models. (a) hypoplasticity, (b) elasto-plasticity. $\sigma_{1}$ and $\sigma_{2}$ are axial and radial stresses respectively. 


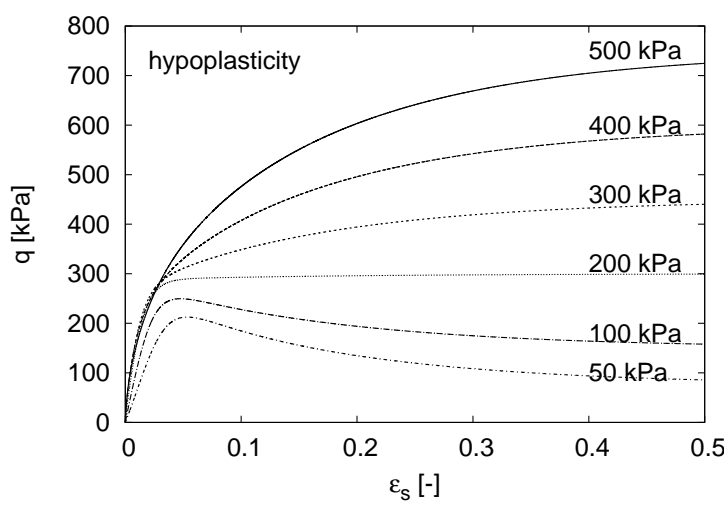

(a)

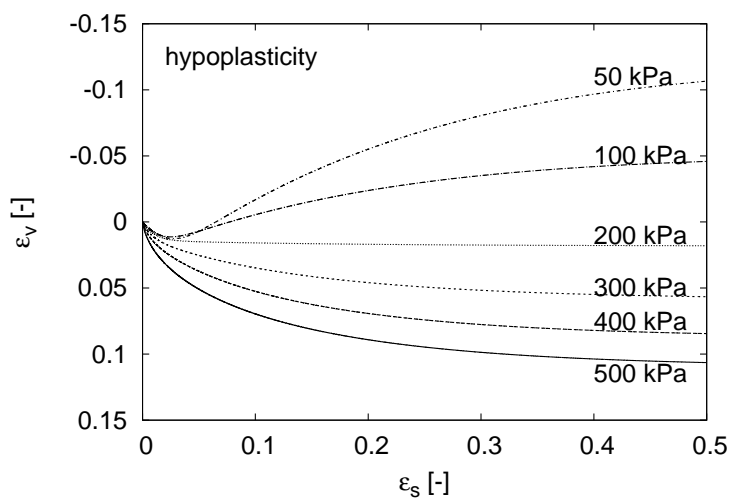

(c)

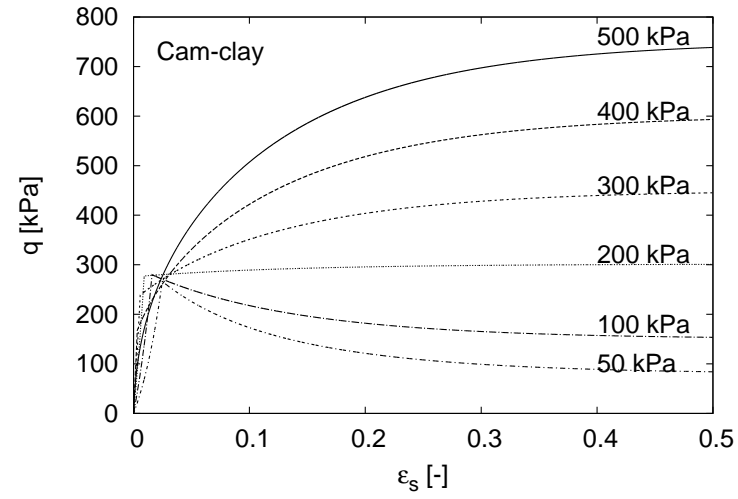

(b)

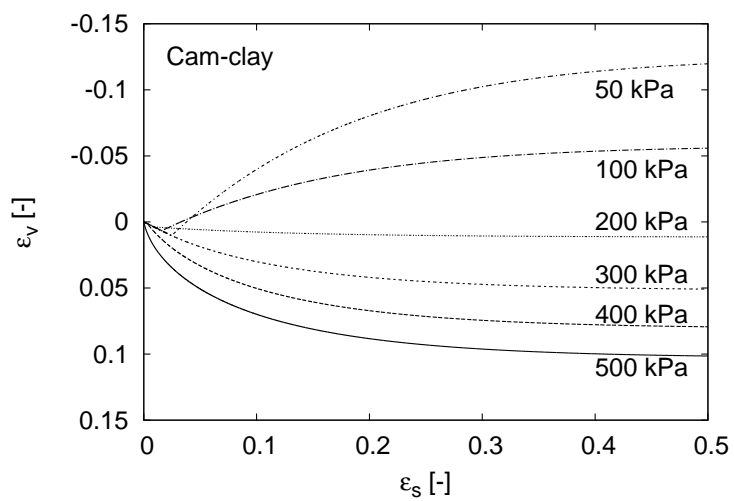

(d)

Figure 2: Predictions of drained triaxial tests for the same void ratio and different radial stresses (labels for cell pressures). 


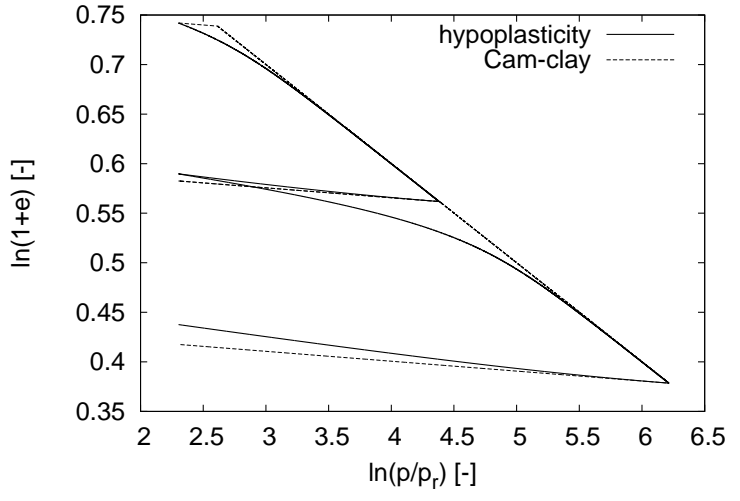

(a)

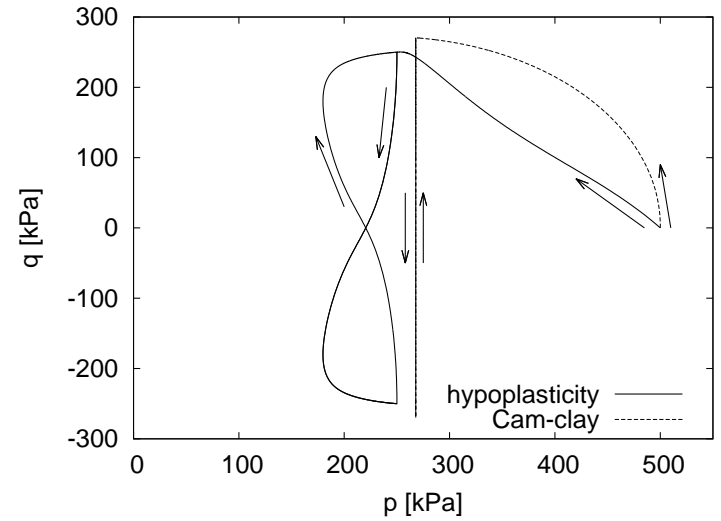

(b)

Figure 3: (a) Isotropic test with several unloading/reloading cycles and (b) cyclic undrained triaxial test. 


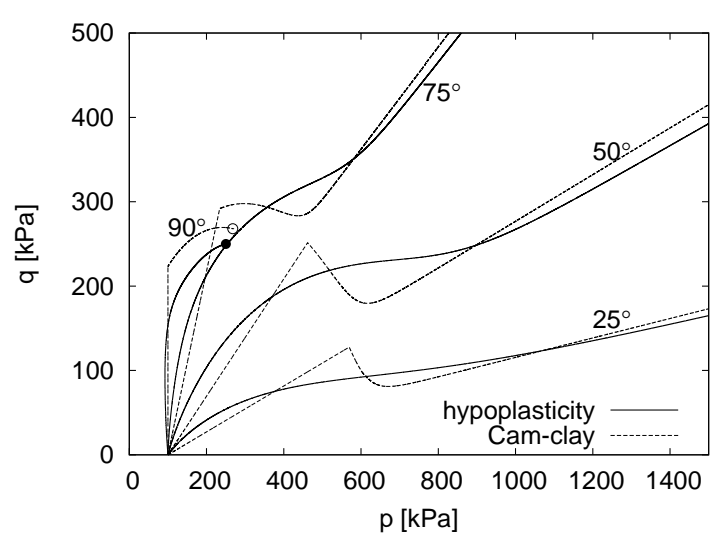

(a)

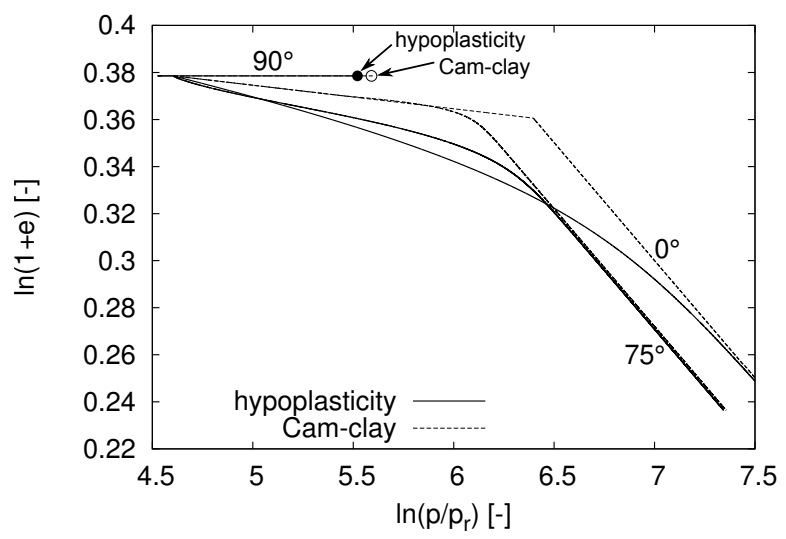

(b)

Figure 4: Proportional compression (constant direction of $\dot{\boldsymbol{\epsilon}}$ ) on initially overconsolidated soil. Indicated are values of $\psi_{\dot{\epsilon}}$, as defined by Gudehus and Mašín (2009). Only selected paths shown in (b) for clarity. 